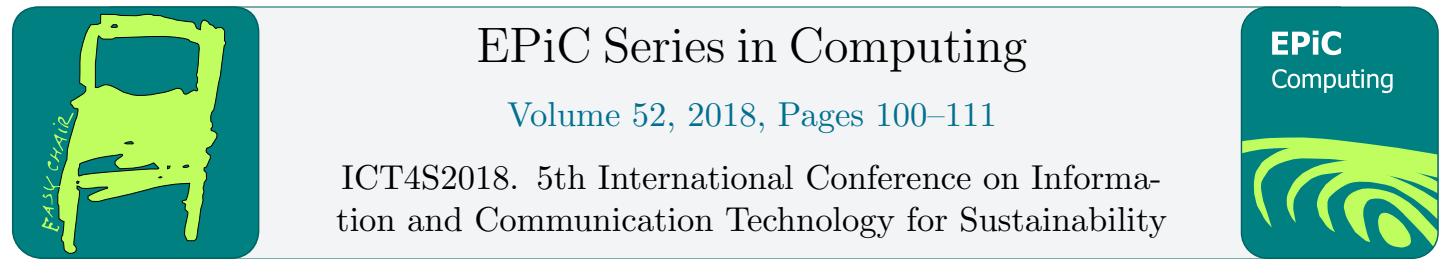

\title{
Envisioning a Community Exemplar for Sustainability in and by ICT
}

\author{
Andreas Fritsch and Stefanie Betz \\ Institute of Applied Informatics and \\ Formal Description Methods (AIFB) \\ Karlsruhe Institute of Technology \\ Karlsruhe, Germany \\ www.s3engineering.org \\ andreas.fritsch@kit.edu, stefanie.betz@kit.edu
}

\begin{abstract}
We understand sustainability as a perspective on the performance of various kinds of systems that puts human well-being in the center of focus. Recognizing that information and communication technology (ICT) is shaping our modern society, there is a need to understand the impact of ICT on sustainability. On the one hand this can be achieved, for example, by extending classic software development approaches to cover sustainability issues. This can be coined sustainability in ICT. On the other hand, innovative ICT approaches offer the potential of directly addressing sustainability issues (sustainability by $I C T)$. Within the ICT4S community, both perspectives are addressed. What is missing, is an overarching perspective that helps to identify interlinkages. In this contribution, we present the case of an online-shop selling ICT hardware products as an community exemplar. We exemplify the usefulness as an overarching example, by relating parts of our existing work on ICT sustainability to it: a process model of the sales process, as well as a representation of sustainability risks related to the sold ICT products. Additionally, we show how ICT4S papers from 2016 can be mapped to the exemplar. We conclude with presenting a community website, where we invite fellow researchers and practitioners to contribute to a growing wealth of sustainability insights.
\end{abstract}

\section{Introduction}

Broadly, sustainability can be defined as the capacity to endure [2]. This shows that long-term thinking and the ability to maintain are key factors of sustainability. Sustainable development is defined as "meeting the needs of the present without compromising the ability of future generations to meet their own needs" [34]. This definition includes a dimension of time, present and future, as well as a direct reference to acknowledging changing requirements of stakeholders. The ultimate goal is human well-being. ICT and Sustainability are deeply interlinked in two ways. On the one hand, ICT (hardware and software) is ubiquitous and has an impact on sustainability, which needs to be analyzed, understood and improved. This can be achieved, for 
example, by extending classic software development approaches (from requirements engineering, to architectural decisions and development) to cover sustainability issues. This "angle of attack" can be coined sustainability in ICT. The application of established methods to analyze sustainability (e.g. life cycle assessment) to ICT products would also fall into this category. On the other hand, innovative ICT approaches offer the potential of directly addressing sustainability issues in new ways - think of intelligent reduction of energy usage through the application of machine learning algorithms, but also the potential of information systems to sustainability by ICT. Within the ICT4S community, both perspectives are addressed by a wealth of approaches, methods and tools [19]. What is missing, is an overarching perspective that provides a platform to combine this work and enables fruitful discussion based on a common ground.

In order to provide a framework to communicate and exemplify the utility of diverse methods and approaches to identify, analyze, estimate and enhance sustainability, we propose an overarching community exemplar. Existing methods and new approaches can be explained and evaluated along this common example. By providing one consistent platform, we expect that this will help to identify potential conflicts or synergies. It can provide a "story" that communicates ideas among practitioners and researchers. As a practical example it can help understand and explain the utility of diverse methods in the context of ICT and sustainability. Given supporting infrastructure, it can also serve as a collection of tools and source of inspiration for analyzing other exemplars in research and practice. Moreover, we envision this exemplar as a basis for teaching materials. For example, small packages of case-material and lecture slides could be provided for either some lessons on specific methods or aspects on sustainability. Additionally, this exemplar could be used in complete courses teaching sustainability and ICT, which eases the understanding of students a lot having one overarching reference. Finally, we think, that at the moment as the awareness on sustainability and ICT is growing and the community is building itself and starts to fly, this is the right moment to introduce an exemplar for sustainability in and by ICT: GoSusy, an online-shop selling ICT hardware products. In the following, we exemplify the usefulness, by presenting some of our existing work on ICT sustainability to the "online-shop story": a process model of the sales process and a model depicting the social sustainability risks of an ICT product (a computer mouse). Additionally, we will present an outline of further approaches that can be adapted to the exemplar. We conclude with describing the concept of a community website and invite fellow researchers and practitioners to contribute.

\section{ICT and Sustainability}

In relation to ICT, two different viewpoints can be found: sustainability in ICT and ICT for sustainability (or sustainability by ICT), where the former is concerned with the principles, practices, and process that contribute to sustainable ICT i.e. maintainability of ICT, resource efficiency of ICT and the latter focuses on ICT to support one or more dimensions of sustainability, concerning issues outside the software systems itself [25]. The Karlskrona Manifesto [3] recognizes both viewpoints of ICT sustainability as an emerging concern and advocates that sustainability must be viewed as a construct across five interdependent dimensions: environmental, economic, individual, social and technical: The economic dimension is concerned with assets, capital and added value that comprises wealth creation, prosperity, profitability, capital investment, income, etc. The environmental dimension encompasses the effects of human activities on natural systems, which include natural ecosystems and resources, climate, pollution and waste, etc. The individual dimension addresses the well-being of humans as individuals, which includes mental and physical well-being, education, freedom, self-respect, mobility, etc. 
The social dimension is concerned with societal communities (groups of people, organizations) and the factors that erode trust in societies, which include social equity, justice, employment, democracy, etc. The technical dimension addresses the concepts of longevity of information, systems, and infrastructure and their adequate evolution with changing surrounding conditions, which includes system maintenance, obsolescence, data integrity, etc. Additionally, the impact on sustainability in these five dimensions manifests itself in three different orders of effect [18]: First order effects appear when ICT are built and used for their direct purpose e.g. the resourcing, manufacturing, installing and usage of the hardware and software needed and sold at the web shop. Second order effects appear when the ICT is used over time from a certain amount of people and this leads to new types of behavior or expectations e.g. users tend to consume more because online shopping is so easy and convenient. Third order effects appear due to a long-term use of the system from a large amount of people e.g. less downtown parking space problems, improved air quality in cities, but also less revenue for the local ICT stores etc. Relationships between some dimensions are immediately visible, but all of these dimensions and intersections need to be analyzed with regard to the impact on the system and its surrounding when designing an ICT system independent from the purpose of the system under development. ICT does also provide the means to proactively improve sustainability (see [18]). Software tools for analyzing and assessing sustainability in diverse domains enable sustainable production patterns. For example, software tools like openLCA ${ }^{1}$ or $\mathrm{Umberto}^{2}$ are used to assess the environmental (and social) impacts of product life cycles. The gained knowledge may then support informed decisions to reduce these impacts. Thinking of information systems in general, ICT does also provide means to communicate sustainability information, such as the results of the aforementioned assessments. By providing transparency for consumers about a product's sustainability characteristics, such kinds of information systems may encourage sustainable consumption patterns (see, as an example for a system for sustainable food supply chains [33]). Finally, new and innovative ICT-related approaches serve as sustainability enablers themselves. Think of the research field of the Smart Grid, where the application of new ICT technologies enables the control and reduction of energy usage and emissions [12].

\section{Related Work}

Exemplars have previously been employed by software engineering researchers as a means to promote understanding among different research groups [13]: in this context, an exemplar is an informal but concise description of a problem representing a real-world task. They may also serve the purpose of comparing methods or to uncover the potential of combining approaches with different strengths and weaknesses. Our idea of an exemplar goes some steps beyond a mere technical purpose. As ICT4S and related communities have a much broader scope, covering not only requirements engineering research, our exemplar is intended to be more inclusive and not focused on one technical problem. The spirit of the exemplar is not of competition, but rather collaboration, like building a mosaic together. ${ }^{3}$ To achieve this, we can learn from other successful initiatives that foster cooperation and communication among researchers: The Open Models Initiative ${ }^{4}$, for example provides a platform to share and comment on conceptual enterprise models. A successful and thriving example for such an online community is

\footnotetext{
$1_{\text {WWw. openlca.org }}$

2 www.ifu.com/umberto/

${ }^{3}$ Not saying that competition should be avoided. Our exemplar could well encompass several more constrained sub-exemplars for specific technical problems in the sense of Feather et al. [13].

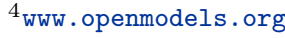


myExperiment, where researchers share experiment workflows and data [8]. Today, the website hosts 10.000 users sharing 2700 of workflows [23]. Another example is CoCoME, a platform that provides a community use case in the area of software engineering. ${ }^{5}$ The platform is designed to support collaboration in empirical research on software evolution by shared knowledge [32]. Interestingly, the CoCoMe platform started to exist in a different form already in 2006 as a Common Component Modelling Example (CoCoME). It has been invented by a Dagstuhl seminar to evaluate and compare existing component models using a practical use case [16].

Furthermore, such an exemplar could not only provide purpose for research, but also for teaching: the idea is also inspired by the case-based learning (CBL) approach and existing online research communities. CBL is a teaching method that uses factually-based, realistic cases for educational objectives. The idea is that the students get on the one hand the possibility to enhance their understanding by getting to see and discuss these cases in the classroom (with the relevant methods and theories applied to them). On the other hand (and even more important) they get the chance to apply methods and theories in complex realistic cases and thus develop their analytical skills [15] [17]. Literature indicates that this kind of learning is very effective [17].

\section{Presentation of Community Exemplar}

The idea of a community exemplar is to combine the strengths of traditional exemplars, casebased learning and online research communities. Initially, we propose a fictional exemplar that can be described by some introductory text, screen shots (like the one given in figure 3), processand goal-models. A first draft of a textual exemplar description can look like this:

GoSusy is an online-shop specialized in selling ICT-products, like desktop-PCs, laptops, peripherals, mobile phones and others. In order to differentiate itself from its competitors, the company has decided to implement sustainable best-practices. They quickly realize that, in order to achieve this goal, they need to take action on various fronts. Also, sustainability in ICT comes with a specific set of challenges. Fair and ecologically labeled products are sold in major supermarket nowadays. But what about a fair labeled computer mouse? What about the energy consumption of the software and servers their infrastructure runs on? How to reduce the carbon footprint of product packaging and transportation? And are the work environments of their employees sustainable? Have they missed some important aspect that may have a negative effect in the long run? Eventually, they realize that they need new tools to analyze their performance with respect to sustainability and new and creative ideas to take action in the right direction.

Later on, this exemplar description may be extended with additional material like multimedia files (e.g. videos of interviews), additional business process models, screen shots, source code, sustainability measurements, energy efficiency measurements of different database configurations, LCAs of products, S-LCAs of products, anything that may help to bring this fictional exemplar to life. ${ }^{6}$

To explain our intuition for developing this specific exemplar as an overarching example for the ICT4S community, we discuss in the following sustainability issues we identified for the online-shop scenario. The intention is to outline the depth and breadth of topics within the ICT4S community that can be related to GoSusy.

\footnotetext{
${ }^{5}$ See https://github.com/cocome-community-case-study/cocome-cloud-jee-platform-migration.

${ }^{6}$ Given a successful launch of the community exemplar, it is even conceivable to make this fiction a reality in the sense of action research.
} 


\subsection{Environmental \& Economic Sustainability Issues}

Due to the extensive use of IT, consumption of electricity is a key indicator of the online sales process sustainability. The optimization of energy consumption can target the company's and/or the customers' use of IT-hardware. On the retailer's side, the main goal is to minimize server load. This can be achieved by various means like simplifying the navigation of the web page, which in turn reduces the number of queries needed to complete an order. An example for implementing this measure would be to combine shopping cart- and completion page into one interface. Another possible improvement is to avoid filling the web pages with a large amount of potentially unnecessary information. On the customer's side, there is room for improving sustainability by shortening the time spent on browsing the web store. Since this might have a negative impact of the company's revenue, it would be beneficial to focus on the average time spent per ordered item. Besides the previously mentioned options, supporting the customer with her choice of products with a helpful customer feedback system etc. could improve this figure.

The delivery process also involves fuel consumption which is caused by shipping orders. An obvious way to mitigate the environmental impact is to use a climate neutral delivery service. For shorter distances, there is also the possibility to use bike courier services. Another resource that is required within the process is packaging material, that can make up for a relatively high amount, especially when many items are packed separately. To tackle this problem, the online shop could encourage its customers to buy more items per order, for instance by offering small discounts for adding items to an order. Furthermore, single sourcing should be considered as it enables the retailer to pack all items into one parcel. The material itself can also be targeted, for example by using only recycled or reusable material. Delivery documents, picking lists etc. need paper as a resource as well. In that regard, it might be beneficial to provide these files in a digital form.

\subsection{Social Sustainability Issues}

As with all online services that require user information, privacy issues are an important topic for an online retailer. Many online stores use their customer's data for monetary purposes such as specifically targeted advertisements. In order to give the user of the web store control over which part of her personal information can be used and which not, it is important to provide detailed privacy options and anonymize data if the user chooses to.

Media reports suggest that employee satisfaction in the online retail industry has been a problem in the past, due to low wages and high pressure to perform (see, e.g. [24]). Although it might have a negative impact on the financial situation of the enterprise, employees should receive a fair wage and be given incentives to perform well. A viable incentive system could provide bonuses for hitting certain picking times for example.

\subsection{Product-related Sustainability Issues}

The sustainability impact of the online shop may not only be attributed to the business activities described above, but also to the sold products. From this perspective, the focus shifts from the internal business processes to the whole product life cycles. Environmental and social issues can be identified at each phase of a product life cycle. Picking just one example, recent studies have described the severe social and environmental impacts that are related to the artisanal mining of cobalt in Congo [31]. 


\section{Sample Material for the Exemplar}

Currently we have already some material available as well as a website to host the community exemplar under the domain www.gosusy.org. Under this domain, we provide a description of the exemplar, supplemented by screen shots and enterprise models. Later on, the website shall provide functionality to upload and share related research artifacts. As a basic structure for the community website, we propose three sections:

- Case Material: This section hosts text, multimedia files and models describing the exemplar. This material can be commented and improved by the community. It is conceivable that in the future, one could compile different configurations of the exemplar (e.g. one "unsustainable" and one "sustainable" online-shop).

- Course Packages: This section hosts bundles of case material and accompanying teaching material like lecture slides and exercises that can be shared within the community.

- Method and Tool Repository: A collection of method and tool descriptions that can be or have been applied to the exemplar. An entry in this collection can either be provided by a link (e.g. to a Github-repository hosting the source-code of a tool) or hosted directly on the site.

In the following we give examples from our previous work and explain how these would fit in the proposed structure.

\subsection{Sample Material: BPMN-Process-Model}

As one example for case material that could be used to enrich the exemplar, we have shared a BPMN-process model of an online sales process on the website ${ }^{7}$. The sheer size of the model is another example, how the proposed online platform may provide benefits to communication among a research community. Within the format of a conference paper we can only give a snippet of the model (see figure 1) and explain it textually, while in an online community it could easily be shared and discussed among researchers. In the following we give a brief description of the shared process model.

The given BPMN-process-model shows the typical procedure of a customer shopping in an online store. It also describes how the store's web server reacts to the users actions and which tasks are performed by the logistics department. The sales-process begins with the user opening the starting page of the online store, which invokes the web server to load the corresponding interface and create a shopping cart object that is used to save items that the user wants to buy. The shopping cart can either be linked to the customer's IP or, if already logged in, to her account. After the starting page has completed loading, the customer moves on to search the desired items and adding them to the shopping cart once found. The server provides the necessary product information by performing a search method on the product database. When the customer wants to finish the purchase, she first opens the shopping cart window to examine its content and then moves on to the completion page. Here the user is asked to login or create a new account. Once the user has submitted the correct login data and confirmed her order, the server stores the order and refreshes the stock amount of the respective items. Afterwards it is analyzed whether products need to be reordered. At the same time, the payment process is executed which results in debiting the customer's bank account/credit card or sending an

\footnotetext{
${ }^{7}$ See https://www.gosusy.org/2018/02/12/bpmn-model/.
} 


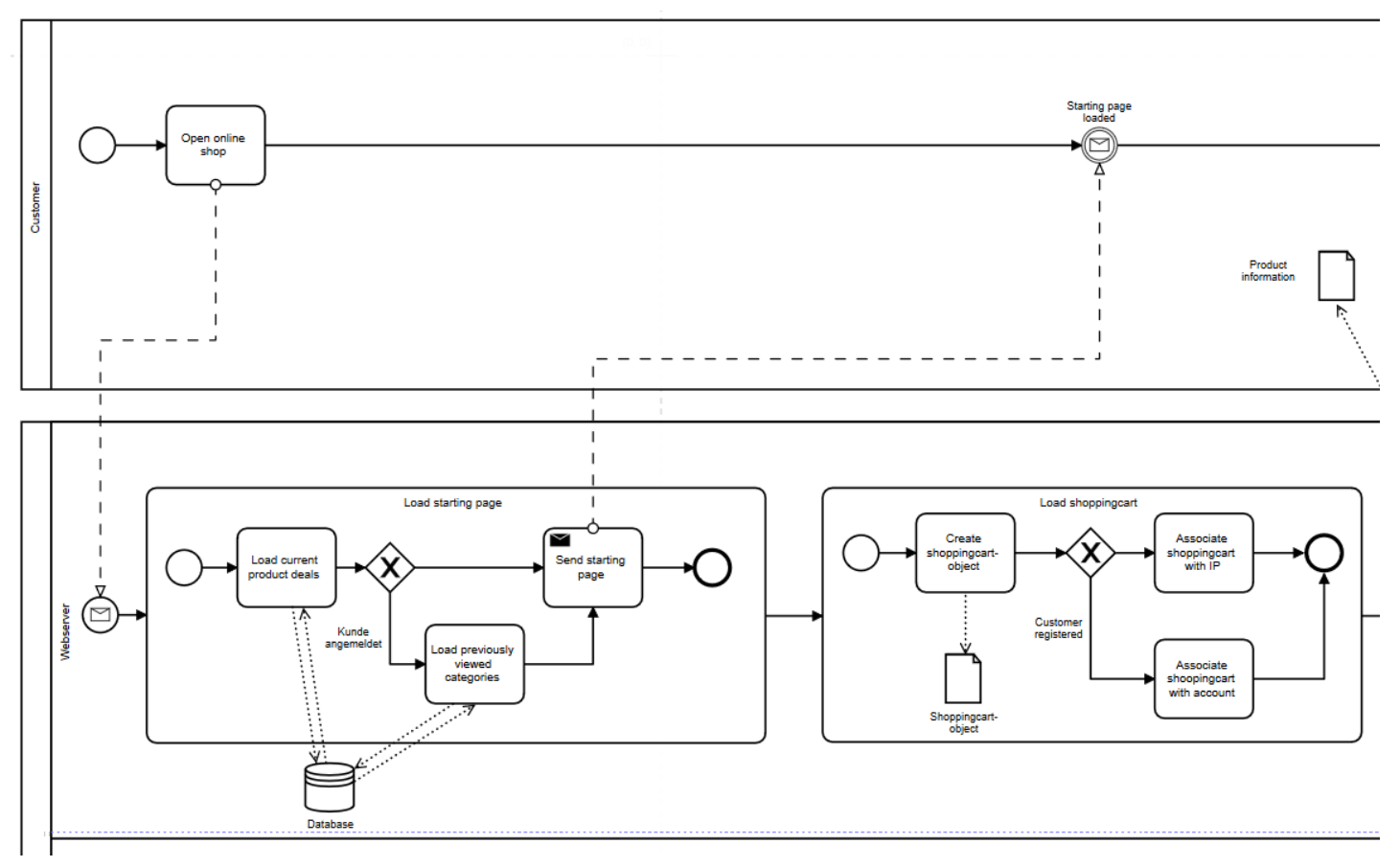

Figure 1: GoSusy example BPMN-Process Model.

invoice. After the payment is completed, the logistics department is informed about the new order and the picking lists are printed. The lists are then used by an order picker to collect the desired items and bring them to a packing station. Then the items are packed up and taken to the shipping area where they are eventually collected by a shipper. Once the customer receives her package she has the option to review the store and the purchased products. Purchased items can also be returned for free within a certain time-frame. To do so the customer applies for the return of one or several items. The server then sends a shipment tag and refunds the price of the returned items. Items that are not returned are brought to book as items sold.

\subsection{Course Samples: Assignments}

We have had two university courses about sustainability and ICT. In this courses the students have produced several assignments around our community exemplar which have been uploaded at the webpage. We have licensed the work under the Creative-Commons Licence and published it in agreement with the students. The areas covered and available are: The concept of sustainability; Data Quality and Availability for S-LCA; Exemplary LCA Study of an ICT product. In addition, we have some course materials available regarding the development of a database for a web-shop including sustainability aspects.

\subsection{Tool: TracyML and Tracy}

We have developed TracyML as a modeling language [4] for providing transparency on a product's social sustainability. The modeling language lays the foundation for Tracy, a web-based tool which allows users to interactively explore a products sustainability characteristics. In 


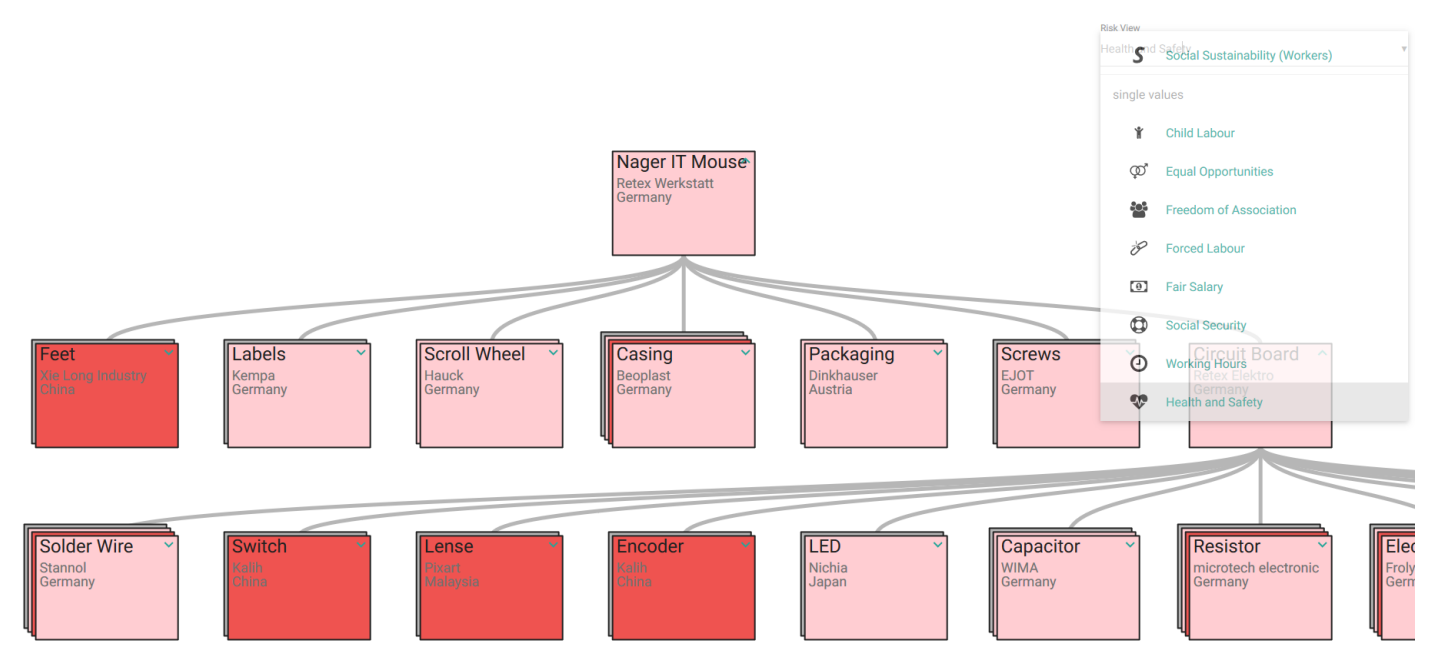

MAP

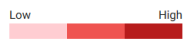

Figure 2: Tracy screenshot.

the context of the webshop, such a tool could be used to provide transparency about the sold products. The screenshot of Tracy shown in figure 2 shows the supply chain of an ICT-product, a computer mouse. The tool visualizes diverse social values, such as the risk of child labour or forced labour within the supply chain. In the context of the webshop, such visualizations could be integrated or linked on the product pages as a means to engage consumers that are concerned with sustainability.

\section{Discussion}

In this section, we first discuss to what extent the proposed use-case could be applicable to the broader ICT4S community. We conclude the discussion with some technical considerations on possible threats that could hinder the development of the use-case as a constructive community platform.

As we have shown in the previous section, the exemplar of an online-shop can be used to exemplify the applicability of approaches in diverse fields like business process modeling on the one hand and social life cycle assessment on the other. What both have in common is that they tackle the challenge of ICT4S, but from different angles. The practical exemplar of GoSusy makes it easy to realize, how both approaches can fit into a bigger picture and could be even used in a concerted manner in order to improve a business's sustainability. In order to analyze, whether the exemplar has a broader potential, not only for our work, but for the whole ICT4S community, we have compiled a list of topics from the contributions to the ICT4S conference 2016. Figure 3 shows a (fictional) screenshot of the GoSusy online-shop. The blue boxes and arrows indicate how the identified topics could relate to GoSusy.

Altogether, 37 papers were published in ICT4S 2016 conference proceedings [14]. We have compiled 10 broader topics from papers and mapped each of the papers to one topic. The 
[GoSusy]

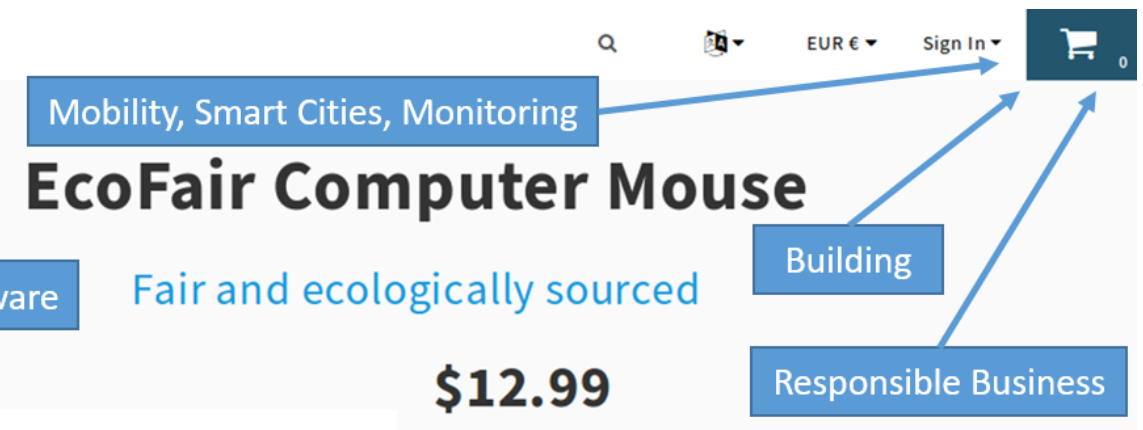

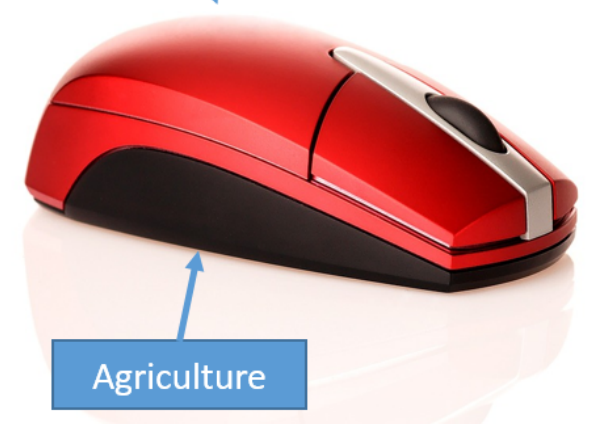

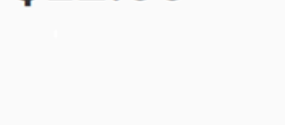

\section{ICT-Hardware}
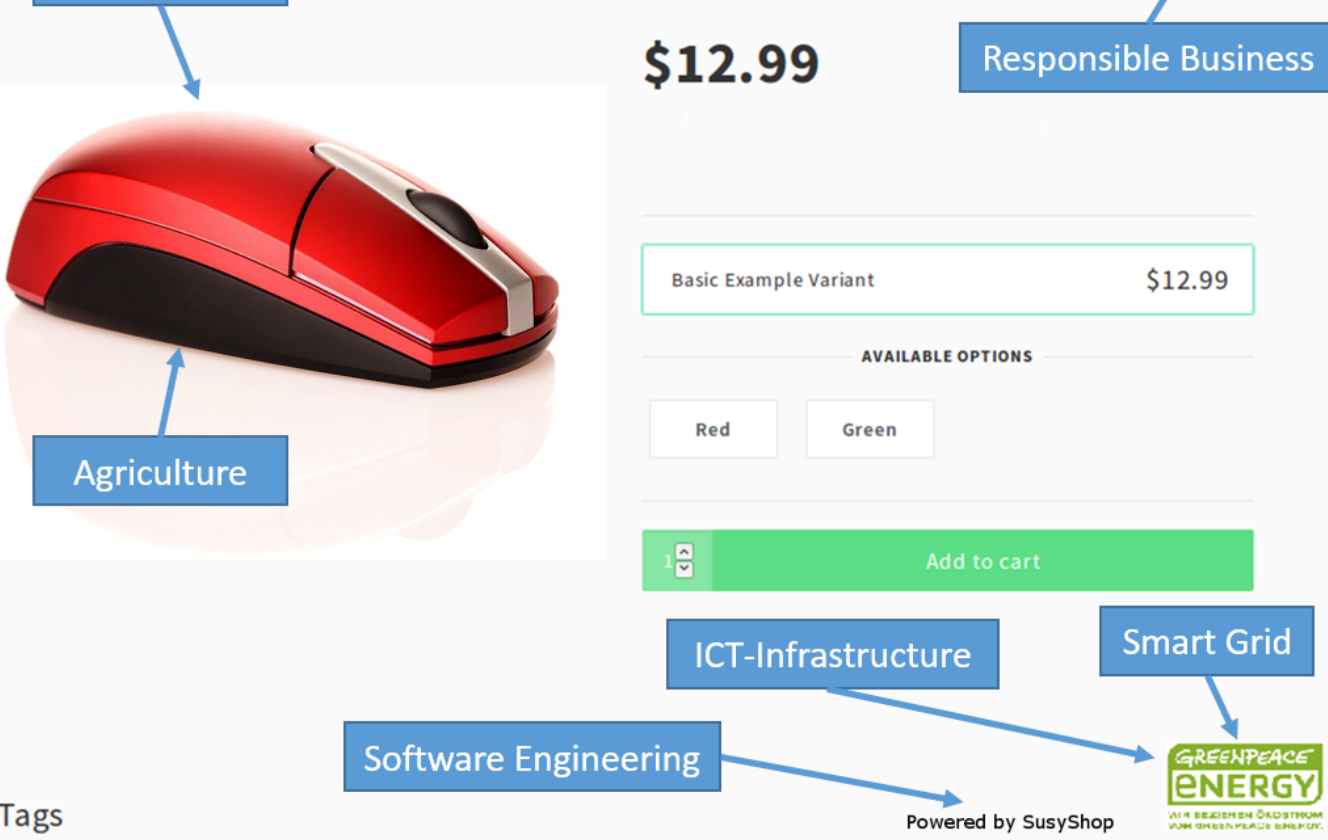

Tags
$\$ 12.99$

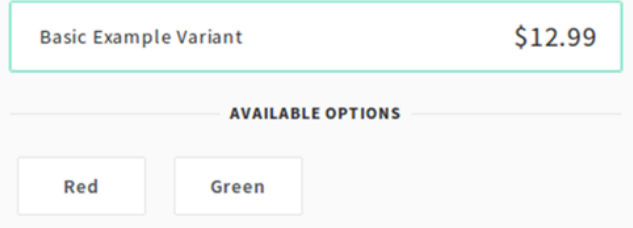

Smart Grid

Figure 3: GoSusy online-shop example screen shot.

final list of topics is: Agriculture, Building, Environmental Monitoring, ICT-Hardware, ICTInfrastructure, Mobility, Responsible Business, Smart Cities, Smart Grid and Software Engineering. A file listing the paper titles, tags and categorization is available for reference under www.s3engineering.org/research. We are fully aware that such broad strokes do not capture the subtleties of each of the research contributions. However, the case in point is that many of the described methods and ideas could be adapted to the exemplar, thus making their relations apparent in a practical example. In the following we give a brief rationale for each topic and how it would apply to the exemplar.

Responsible Business: Research concerned with the question how a business in general could behave more responsible [21] could lay a foundation to GoSusy's business practices (in the figure represented by the shopping cart).

Software Engineering: All of the research addressing sustainable software engineering (e.g. [1]) would influence the software the online shop runs on (in the example screen shot a fictional software "SusyShop").

ICT-Hardware: Papers like [11] and [10] are concerned with ICT-product sustainability. The methodology applied here, (Social) Life Cycle Assessment, would be relevant for GoSusy to analyze the sustainability of the sold products. 
ICT-Infrastructure: Research addressing the energy consumption of data centers [27] or internet architecture [9] are relevant for analyzing and improving the ICT infrastructure (e.g. servers) of the online-shop. In the example screen shot, we have mapped the topic to a label claiming that the online-shop servers use sustainable energy.

Smart Grid: The energy infrastructure affects the ICT-infrastructure, as discussed for example by [22], and energy usage of the company.

Building: Results and findings from Smart Home [30] and Building Management Systems [28] research could be useful to improve energy efficiency of the workplace for GoSusy's employees or the warehouses, where the products are stored. In the figure we have mapped the topic to the shopping cart meaning that the topic is relevant for the business processes of an online store.

Agriculture: At first sight, research addressing sustainable food systems [29] is not related to an online shop selling ICT-Products. Yet, supply chains for ICT-products may well interrelate with food production, as, for example, casings could be made from bio-plastics (see, e.g. [6]).

Mobility: Research on sustainable mobility [5] may address the commuting of GoSusy's employees and logistics.

Smart Cities: GoSusy as a working place or the utilized warehouses are part of a bigger ecosystem that is addressed by research on Smart Cities (e.g. [20]).

Environmental Monitoring: Environmental monitoring of gas leakages [7] or water quality [26] affect working spaces and warehouses. The last three topics all interelate with the online store's busines processes and are mapped to the shopping cart in the screen shot.

As this brief discussion shows, all the identified topics appear to be relevant for GoSusy. It seems viable that the discussed approaches and results can be related to the proposed community exemplar. Concerning the technical viability of GoSusy as a online community platform, there is a threat of possible "bad material" that becomes part of our community exemplar. One possibility to minimize the threat of low quality material is to only publish peer reviewed material. But, we think this minimizes the possibilities of the exemplar, as for example also white papers and teaching material should be included. Another option to minimize the threat is to have a core team checking the submitted data, which may be too much work (hopefully someday and also not within the capabilities of the team). Another option is to tag the data pointing out if it is based on peer reviewed material or not, in order to provide the possibility for each user to decide what data to use and how. Currently, we are planning to use the last suggested version and tag the data. Additionally, we are planning to provide the possibility for the user to comment on the quality of the available material.

\section{Summary and Outlook}

In this paper we have presented a vision of a community exemplar based on a web shop. We have already established a webpage (www.gosusy.org) and several artifacts concerning the exemplar. We have collected simulation data concerning the sales process, several process models, a social LCA and goals models. In addition, we are providing the platform to connect and further gradually enrich the exemplar. In its current state, it provides the functionality for researchers to submit and discuss material and links in the categories case material, course packages and method and tool repository. We would like to emphasize that one advantage of the idea is, that it allows gradual adoption. It could only be mentioned in a conference talk to quickly communicate the general purpose of an approach - or even implemented as a real-life web shop to enable action research. Ultimately we envision to actually run the GoSusy online shop. This running instance of the exemplar would reflect and implement the identified best practices of 
sustainability in and by ICT. As such, it would provide an additional means to evaluate the usefulness of the collected tools and approaches. Moreover, with the online shop up and running we enable additional analysis of consumer behaviour with regard to sustainability topics. We are aware that this is just a start, but we would like to initiate the discussion and invite the ICT4S community to contribute.

\section{References}

[1] Maryam Al Hinai and Ruzanna Chitchyan. Engineering requirements for social sustainability. In Proceedings of ICT for Sustainability 2016. Atlantis Press, 2016.

[2] Christoph Becker, Stefanie Betz, Ruzanna Chitchyan, Leticia Duboc, Steve M Easterbrook, Birgit Penzenstadler, Norbet Seyff, and Colin C Venters. Requirements: The key to sustainability. IEEE Software, 33(1):56-65, 2016.

[3] Christoph Becker, Ruzanna Chitchyan, Leticia Duboc, Steve Easterbrook, Martin Mahaux, Birgit Penzenstadler, Guillermo Rodriguez-Navas, Camille Salinesi, Norbert Seyff, Colin Venters, Coral Calero, Sedef Akinli Kocak, and Stefanie Betz. The karlskrona manifesto for sustainability design. arXiv preprint arXiv:1410.6968, 2014.

[4] Stefanie Betz, Andreas Fritsch, and Andreas Oberweis. Tracyml - a modeling language for social impacts of product life cycles. In C. Cabanillas, S Espana, and S Farshidi, editors, Proceedings of the ER Forum 2017 and ER 2017 Demo track, Valencia, 2017.

[5] Dominik Bucher, Francesca Cellina, Francesca Mangili, Martin Raubal, Roman Rudel, Andrea Emilio Rizzoli, and Omar Elabed. Exploiting fitness apps for sustainable mobility - challenges deploying the goeco! app. In Proceedings of ICT for Sustainability 2016, 2016.

[6] Kevin Bullis. Cheap plastic made from sugarcane. MIT Technology Review, 2011.

[7] Vlad Coroama and Mattias Hjer. Assessing ghg benefits induced by ict services in practice: A case study and resulting challenges. In Proceedings of ICT for Sustainability 2016. Atlantis Press, 2016.

[8] David De, Roure Carole, and Goble Robert Stevens. The design and realisation of the myexperiment virtual research environment for social sharing of workflows. Future Generation Computer Systems, 25:561-567, 2009.

[9] Etienne-Victor Depasquale and Chris Preist. A case for the development of data standards for reporting projections of growth in the internet's energy consumption. In Proceedings of ICT for Sustainability 2016. Atlantis Press, 2016.

[10] Elisabeth Ekener and Elena Mokeeva. Mobile phones: social impacts during the use phase. In Proceedings of ICT for Sustainability 2016. Atlantis Press, 2016.

[11] Mine Ercan, Jens Malmodin, Pernilla Bergmark, and Emma Kimfalk andEllinor Nilsson. Life cycle assessment of a smartphone. In Proceedings of ICT for Sustainability 2016. Atlantis Press, 2016.

[12] Hassan Farhangi. The path of the smart grid. IEEE power and energy magazine, 8(1), 2010.

[13] Martin S. Feather, Stephen Fickas, Anthony Finkelstein, and Axel van Lamsweerde. Requirements and specification exemplars. Automated Software Engineering, 4(4):419-438, Oct 1997.

[14] Paola Grosso, Patricia Lago, and Anwar Osseyran, editors. Proceedings of ICT for Sustainability 2016, volume 46 of Advances in Computer Science Research, 2016.

[15] Martin Henkel, Ilia Bider, Erik Perjons, Filip Mårtensson, and Martin Zainali. Reusing cases for teaching enterprise modelling-feasibility study and reality check. 5th Symposium on Conceptual Modelling Education (SCME 2017), 2017.

[16] Sebastian Herold, Holger Klus, Yannick Welsch, Constanze Deiters, Andreas Rausch, Ralf Reussner, Klaus Krogmann, Heiko Koziolek, Raffaela Mirandola, Benjamin Hummel, et al. Cocome-the common component modeling example. Lecture Notes in Computer Science, pages 16-53, 2008. 
[17] Clyde Freeman Herreid. Because wisdom can't be told: using case studies to teach science. Peer Review, 7(2):30, 2005.

[18] Lorenz M Hilty and Bernard Aebischer. Ict for sustainability: An emerging research field. In ICT Innovations for Sustainability, pages 3-36. Springer, 2015.

[19] Lorenz M. Hilty and Bernard Aebischer. ICT innovations for sustainability, volume 310. Springer, 2015.

[20] Anna Kramers, Josefin Wangel, and Mattias Hjer. Governing the smart sustainable city: the case of stockholm royal seaport. In Proceedings of ICT for Sustainability 2016. Atlantis Press, 2016.

[21] Maria Mora-Rodriguez and Chris Preist. The role of interoperability in sustainability decisions: the case of cdp. In Proceedings of ICT for Sustainability 2016, 2016.

[22] Jordan Murkin, Ruzanna Chitchyan, and Alastair Byrne. Enabling peer-to-peer electricity trading. In Proceedings of ICT for Sustainability 2016. Atlantis Press, 2016.

[23] MyExperiment. About, 2017. accessed 19.11.2017 19:30.

[24] NBC. The future of retail work: Many see low pay, little flexibility, 2014. accessed:

[25] Birgit Penzenstadler and Henning Femmer. A generic model for sustainability with process- and product-specific instances. In 2013 workshop on Green in/by software engineering, pages 3-8, New York, 2013. ACM.

[26] Ivan S. Razo-Zapata, Giacomo Piovan, and Christian Penny. Watergram: A citizen-centric platform to monitor water quality. In Proceedings of ICT for Sustainability 2016. Atlantis Press, 2016.

[27] Daniel Schlitt and Wolfgang Nebel. Data center performance model for evaluating load dependent energy efficiency. In Proceedings of ICT for Sustainability 2016. Atlantis Press, 2016.

[28] Paul Shabajee, Chris Preist, Daniel Schien, John Brenton, and Chris Jones. Diagnostic visualisation of building management system energy data. In Proceedings of ICT for Sustainability 2016. Atlantis Press, 2016.

[29] Asa Svenfelt and Jorge Luis Zapico. Sustainable food systems with ict. In Proceedings of ICT for Sustainability 2016. Atlantis Press, 2016.

[30] Seyed Amin Tabatabaei. A data analysis approach for diagnosing malfunctioning in domestic space heating. In Proceedings of ICT for Sustainability 2016. Atlantis Press, 2016.

[31] Nicolas Tsurukawa, Siddharth Prakash, and Andreas Manhart. Social impacts of artisanal cobalt mining in katanga, democratic republic of congo. Öko-Institut eV, Freiburg, 2011.

[32] Birgit Vogel-Heuser, Thomas Simon, Jens Folmer, Robert Heinrich, Kiana Rostami, and Ralf Reussner. Towards a common classification of changes for information and automated production systems as precondition for maintenance effort estimation. In Industrial Informatics (INDIN), 2016 IEEE 14th International Conference on, pages 166-172. IEEE, 2016.

[33] PM Nel Wognum, Harry Bremmers, Jacques H Trienekens, Jack GAJ van der Vorst, and Jacqueline M Bloemhof. Systems for sustainability and transparency of food supply chains-current status and challenges. Advanced Engineering Informatics, 25(1):65-76, 2011.

[34] World Commission on Environment and Development. Our Common Future. available from undocs.org/A/42/427, 1987. 